\title{
COMPARATIVE STUDY OF THREE DIFFERENT TECHNIQUES FOR BROKEN INSTRUMENT REMOVAL
}

\author{
Mennattullah Mohsen Hafez ${ }^{*}$, Ahmed Mostafa Ghobashy ${ }^{* *}$ and Ehab Elsaied Hassanein ${ }^{* * *}$
}

\begin{abstract}
The purpose was to evaluate three systems for broken instrument retrieval (Ultrasonic, Instrument Retrieval System "IRS", and Microtube + Cyanoacrylate Adhesive). Cross-sectional area, time, and perforation incidences were recorded. A total of 60 extracted molar teeth were used. Teeth were placed in a cast compound arch shaped mold to facilitate handling. Teeth were divided into two groups according to type of motion used Group I: Reciprocating motion $(n=30)$ Group II: continuous rotation motion $(n=30)$, and three subgroups according to the retrieval instrument used. Concerning retrieval time, Ultrasonics showed least time needed for retrieval in comparison to IRS and Microtubes. Ultrasonics can be used as universal retrieval systems for canal obstructions.
\end{abstract}

KEY WORDS: IRS, microtube, Retrieval, ultrasonics, Time.

\section{INTRODUCTION}

Endodontic treatment is an important requirement in the dental field. The success and survival rate of a permanent dentition after endodontic treatment is determined by the ability to ensure complete debridement of canals and ability to provide adequate proper seal with no obstructions affecting the overall outcome. Recently, the newer techniques for cleaning and shaping using rotary files rendered the process of instrumentation quick and more consistent. However, the procedural errors resulting from this technology sometimes cannot be prevented. An instrument will fracture if its ultimate strength is exceeded, or if a crack has extended to such a degree that the remaining cross-section of material is unable to withstand the usual operating load ${ }^{(1)}$. Some of the factors, that influence the broken instrument removal technique, are the root canal anatomy, the length of the root, the type of instrument broken, angle and radius of curvature ${ }^{(2)}$. Instrument fracture hinders the clinicians from optimal preparation, and renders them curious about what they have to do next. They have to evaluate the treatment options with consideration of pulp status, root canal configuration, anatomy, position, and type of fractured segment. The visualization of broken fragment with the dental operating microscope aided in the successful retrieval

\footnotetext{
* Assistant Lecturer, Department of Endodontics, Misr International University.

** Lecturer, Department of Endodontics, Misr International University.

*** Professor, Department of Endodontics, Misr International University.
} 
attempts ${ }^{(4)}$. There is no any standardized procedure for safe and consistently successful removal. The results are usually subjective to operator skills and armamentarium. Advancing a file inside a canal will result in screwing of the file and any effort to remove it will lead to breakage, especially in curved canals. Certain methods were used for removal of separated instruments in straight and curved canals.

\section{MATERIALS AND METHODS}

\section{Specimen preparation}

A Total of sixty extracted human mandibular molars were used in this study. All molars were with mature apices and normal root morphology. Angle and radius of curvature for the mesial roots were recorded(5).

\section{Storage of specimens}

Samples were washed under running water, air dried, cleaned and sterilized. Kept in sodium hypochlorite $\mathrm{NaOCl} 5 \%$ for one hour and then, stored in normal saline till time of use.

\section{Preparation of samples}

Teeth were flattened with a cylindrical stone and high-speed hand piece to obtain mesial roots with average length of $13 \mathrm{~mm}$. Negotiation and patency were ensured with a \#10 k-file. Sodium hypochloride solution $(\mathrm{NaOCl})$ was used as an irrigating solution. Pre-instrumentation radiographs were taken.

\section{Placement of the Roots in Mold}

Roots were placed in a cast compound arch shaped mold with 14 roots each. This mold was used to ensure reproducibility of results and to ensure that the pre and post radiographs are the same (6).

\section{Sample Grouping}

The sixty samples were divided into two equal groups according to instrumentation system as follows:
Group I: Instrumented with reciprocating file system $(n=30)$

Group II: Instrumented with Continous Rotating file system $(n=30)$

Each group was further subdivided into three equal subgroups ( $\mathrm{n}=10$ each):

Subgroub IA, IIA: Retrieved using Ultrasonic $(\mathrm{n}=20)$

Subgroub IB, IIB: Retrieved using IRS (n=20)

Subgroub IC, IIC: Retrieved using Microtube and adhesive systems. $(\mathrm{n}=20)$

\section{Root canal preparation before instrument separation}

Group I: The canal was filled with Glyde then; preflaring was done until binding point and file $\# 10$, and \#15 k files were used to create a glide path for wave one file according to manufacturer's instructions. The WaveOne small 20.06 file was used to prepare the canal. The file was removed and wiped clean. Irrigation was done and the file was introduced again in the canal until the working length was reached. A reciprocating motor "SatelecI endo" and a 6:1 reducing handpiece were used. The counter clockwise $(\mathrm{CCW})$ rotation which engages dentine is greater than the clockwise $(\mathrm{CW})$ rotation which disengages dentine. The $\mathrm{CCW}$ rotation is 150 , the $\mathrm{CW}$ rotation is 30

Group II: Protaper Universal Series Rotary Nickel Titanium System, X-Smart continuous rotation motor and a 16:1 handpiece were used at a speed of 300r.p.m. and torque was adjusted with each file according to the manufacturer instructions. The sequence as recommended by the manufacturer using a crown down technique was the S1 and S2 Shaping files prepared the coronal two thirds of the canal and moved apically. Proper irrigation was done, then S1, S2 files were used back to the full working length. After the preparation of the coronal two thirds, the finishing file F1 was used after the 
canal was filled with irrigating solution, to prepare the apical one third.

\section{Instrument Separation inside the root canal:}

Instruments were notched with a fine tapered diamond stone to facilitate file separation at a set length $5 \mathrm{~mm}$ from the tip inside the MB canal and to ensure reproducibility of results. Rotary notched instruments were run with a high torque handpiece to break the instrument at the predetermined place inside the canal. In group I Wave One file 0.25 tip diameter and 0.08 taper in a reciprocating motion was broken inside the MB canal. While in group II F2 file 0.25 tip diameter and 0.08 taper in a continuous rotation motion was broken inside the MB canal. A periapical radiograph was taken to the samples after file separated inside the canal.

\section{Attempts to remove broken file:}

Under the magnification of Dental operating microscope in a moderate magnification range offered magnification from $8 \mathrm{x}$ to $16 \mathrm{x}$, a straight line access was created using Gates Glidden burs whose maximum cross sectional diameter was slightly larger than the visualized fragment in a brush like manner acting as a staging platform removing circumferential root dentin around and above the broken fragment till the coronal tip was exposed. To use the maximum diameter of the drill and create end cutting- drill, the drill was modified by cutting its guiding tip off its maximum crosssectional diameter perpendicular to its long axis by a high speed flat fissure diamond bur. The modified Gates Glidden was carried into the pre-enlarged canal, creating a staging platform, trephining dentin coronal to the fragment.

Subgroup A: Ten teeth in each group were retrieved using Ultrasonic Method. Direct visualization was attempted using dental operating microscope. P5 newtron ultrasonic device was used at 4-6 power. Water coolant was used to prevent heat generation.. The ET25 Ultrasonic tips mounted in an ultrasonic scaler were used wedged between dentinal wall and separated fragment and activated within the lower power settings and moved lightly in a counter-clockwise direction Vibration continues till the fragment was successfully removed.

Subgroup B: Ten teeth in each group were retrieved using the iRS method. Straight line access was done to expose the coronal portion of fragment. A Gates Glidden drill was inserted after modifying the tip as previously mentioned to create a staging platform. Afterwards an E7 ultrasonic tip was inserted inside the MB canal and vibrated in a counter-clockwise direction exposing the coronal $2 \mathrm{~mm}$ of the separated instrument. The broken file laid against the outer wall of the canal, so, the red handle IRS 21 gauges $(0.80 \mathrm{~mm})$ - the one used in this study- was inserted with the long part of its beveled end towards the outer wall and scooped out the head of the broken fragment and guided it towards the microtube. Once the microtube had been positioned, the same color coded screw wedge was inserted and slided internally through the microtube's length until it contacted the obstruction. The screw wedge was turned in a counterclockwise (CCW) direction for few degrees until tightened, wedged, and displaced the fragment. Then the fragment, microtube, and screw wedge were removed out of the canal.

Subgroup C: Ten teeth in each group were retrieved using the microtubes and adhesive method. A straight line access to the separated file was created using the same maneuver as before. The 20 gauge microtube was inserted into the canal over the coronally exposed part of the fragment and completely tightened over it. A Cyanoacrylate adhesive was then dropped to the inside of the tube, kept in place for 3 minutes to ensure proper adhesion of the whole assembly, rotated in a counterclockwise direction, and then withdrawn outside of the canal ${ }^{(7,8)}$. 


\section{Methods of evaluation}

\section{A- Cross sectional area}

Teeth mounted in the cast compound arch shaped mold placed on scanner of the i.Cat CBCT imaging system, each mold was scanned with a voxel dimension 0.125 for pre and post retrieval. A three dimensional image of pulp space was formulated and the cross sectional area of circumferential dentin was digitally recorded at different reading levels from the apex $(5 \mathrm{~mm}, 7 \mathrm{~mm})$. Pre and post retrieval readings were calculated through the software (In vivo Viewer). Data were recorded by different examiners and mean value was taken to prevent any bias. Fig (1)

\section{B-Time needed for retrieval}

A stop watch was set from the beginning of the attempts to record the time needed to successfully remove the broken file. The least time a technique needed would be considered as the most successful way of retrieval

\section{C-Perforation incidences}

Any change in the integrity of the canal was recorded and any perforation incidences that occurred was photographed and recorded.

\section{RESULTS}

Statistical analysis was performed with IBM ${ }^{\circledR}$ SPSS® Statistics Version 20 for Windows

3.1- Cross sectional area: Percentage of Increase in cross-section area of circumferential dentin:

In specimens prepared with Protaper system and retrieved with Ultrasonics, IRS as well as Microtube, there was no statistically significant difference between different root levels.

In specimens prepared with WaveOne system and retrieved with Ultrasonic system, there was no statistically significant difference between the root

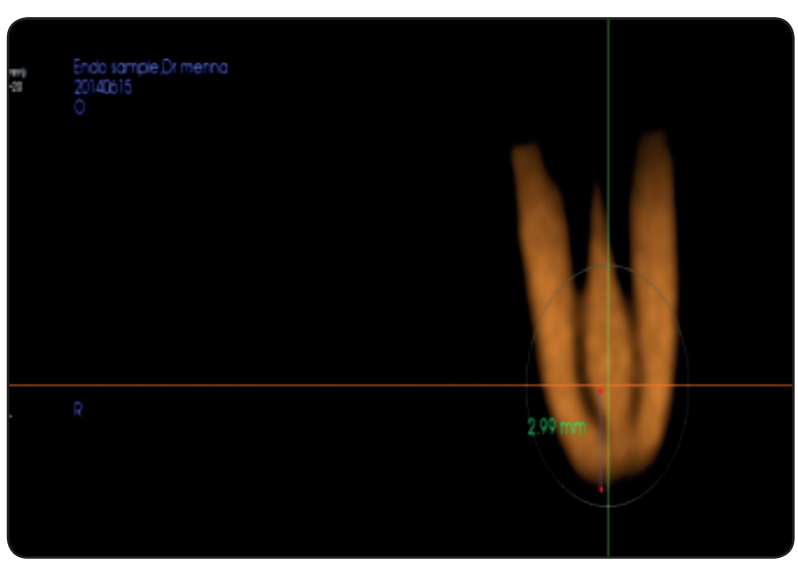

Step (1) drawing a line from the root apex till level of measurement

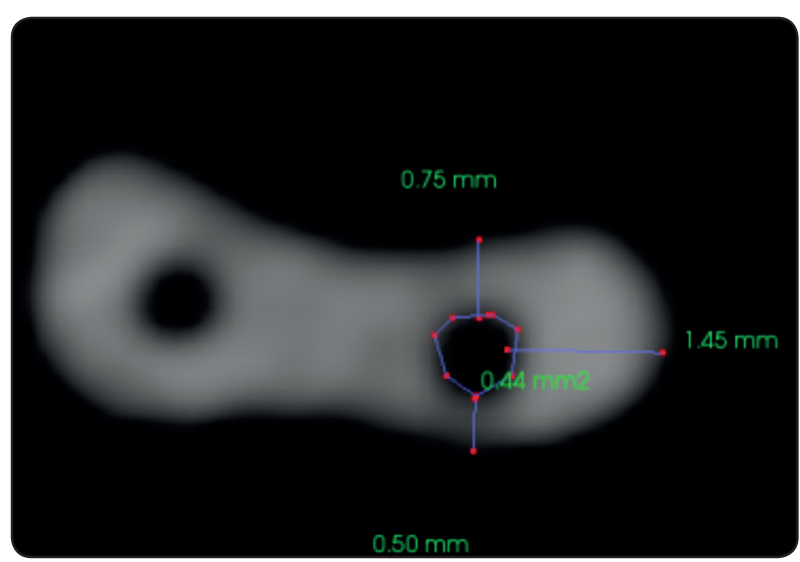

Step (2) Cross sectional area of circumferential dentin measures.



Step (3) Post retrieval images, choosing level at which cross sectional area will b measured a: at $5 \mathrm{~mm}$, b: at $7 \mathrm{~mm}$.

Fig. (1) 
levels. While in specimens retrieved with IRS, $7 \mathrm{~mm}$ level showed the statistically significantly highest mean $\%$ increase in cross-section area. $5 \mathrm{~mm}$ level showed the statistically significantly lowest mean \% increase in cross-section area. Regarding specimens retrieved with Microtube, there was no statistically significant difference. At $7 \mathrm{~mm}$ levels it showed the statistically significantly highest mean $\%$ increase in cross-section area while, $5 \mathrm{~mm}$ level showed the statistically significantly lowest mean \% increase in cross-section area.

\section{Retrieval time}

\section{Effect of retrieval system}

There was no statistically significant difference between mean retrieval times in specimens retrieved with IRS and Microtube systems; both showed the statistically significantly highest mean times. US showed the statistically significantly lowest mean retrieval time. Since the interaction between the two variables (preparation and retrieval systems) is non-statistically significant, so the same results are obtained with each preparation system.

\section{Perforation}

In specimens retrieved with US retrieval system, all specimens showed no perforation. In specimens retrieved with IRS as well as Microtube retrieval systems, there was no statistically significant difference between prevalence of perforation after using the two preparation systems.

\section{DISCUSSION}

Instrument separation initiates a foothold due to accidental separation of the apical end of rotary nickel titanium instruments. Although separated files in root canals did not always result in an unfavorable prognosis, instrument fracture definitely impeded the microbial control ahead of the obstruction and complicated the process of endodontic treatment ${ }^{(9)}$. Retrieving an instrument fragment in a canal might cause excessive removal of dentin structure, decreased root strength, and even perforation. However, due to technological breakthrough in the field of endodontics especially in vision, ultrasonic instrumentation, and microtube delivery methods, separated instruments could usually be retrieved ${ }^{(10-12)}$.

Nickel-Titanium instruments are more susceptible to fracture with $78.1 \%$ of separation ${ }^{(13)}$. That's due to the low tensile and yield strength which can easily turn the endodontic appointment into a detrimental one. ${ }^{(14,15,16)}$

This study was designed to evaluate the appropriate method to retrieve a separated instrument in continuous rotation motion and reciprocating motion. Those two types were chosen as for the rotation motion being the most common motion among most of the rotary systems present in the market. Reciprocation motion being the least motion that allow engagement of file to dentinal wall so decrease the wedging and locking action and decrease fracture incidence of file ${ }^{(17-20)}$. All samples were embedded in a cast compound mold as these molds provide repetitive placement of specimens, facilitating pre and post retrieval CBCT scan. They ensure reproducibility of results and allow multiple removals for convenience and reorientation of the samples during replacing in the exact position of each sample $e^{(7,21)}$.

Under magnification, F2 and Primary files were intentionally weakened by a diamond stone creating a notch at $5 \mathrm{~mm}$ from tip to ensure consistent results and that all the files will be separated at the same place. Using the dental operating microscope during instrument retrieval was of prime importance to avoid undue removal of the remaining dentin thickness and further weakening of the root. So, the 1 and 1.6 magnification offered a moderate magnification range of the image from $8 \mathrm{x}$ to $16 \mathrm{x}$. This provided superior quality, high optical clarity, enhanced vision and high resolution. It aided in the preservation of tooth structure. This came in 
agreement with what was proposed by Alomairy ${ }^{(22)}$ that the perfect combination between Ultrasonics and microscope in the field of endodontics played a crucial role in increasing successful removal of fractured instruments from narrow curved canals. The direct vision and increased illumination inside the canal aided in the visualization of the intracanal obstruction locating its position allowing Ultrasonics to work safely away from furcations, and stay centered ${ }^{(23,24)}$.

Files were chosen to be fractured in curved mandibular molar teeth, which, was the common place for broken files. As described by Wu et al ${ }^{(16)}$ separation incidences in molars are higher than that of premolars and anterior. Also, they assumed that separation is very common in mesial canals than any other place. This finding agrees with that of Shen et al ${ }^{(9)}$, Cuje et al ${ }^{(25)}$.

Ultrasonics was selected for being the gold standard instrument used and for the universal availability. It was used as a kind of control group and comparison was then carried out between the three systems in order to evaluate the effectiveness of the other two systems. Ultrasonic devices have been advocated for the removal of broken instruments, because the tips can be used deep in the root canal system providing constant visualization of the energized tip. With greater incidence of rotary instruments' fracture ultrasonic vibration was needed to dislodge the fractured fragment and make it sufficiently loose to be removed ${ }^{(7)}$. Dry field was recommended when using ultrasonic tips for negotiation, ensure constant vision and to enhance visualization of the fragment ${ }^{(8,22)}$. This came in agreement with the questionnaire proposed by Madarati et al ${ }^{(7)}$. They stated that $56 \%$ worked in a dry field, while $21 \%$ always used irrigation. However, Plotino et al ${ }^{(26)}$ reported that this caused brown discoloration and it increased the heat generation from the tip to the root dentin. So the use of internal irrigation tips was used in this study to allow for better cutting efficiency and to decrease heat generation.

Many of the microtube systems required excessive removal of $\operatorname{dentin}^{(8,22)}$. The critical distinction when considering microtube was not the inside diameter of the device, but it is the outer diameter that indicates how deep it could be safely introduced inside the canal, and how successful it could be ${ }^{(27-29)}$. The tube mechanics dictates the engagement of the file to a hole inside the tube. This can be explained as if we divided the root into two parts: part A: above the curve, part B: below the curve. Regarding the fragment, were broken at the curve or below the curve, the challenge was to create a spill way for the file to exit with the long axis of part A "straight Part of the canal". When the tube enters to engage the fragment it wedges it and locks it in its place preventing this free movement subsequently, failure of retrieval. This came in agreement with Hulsmann ${ }^{(30)}$ and Wilcox et al where they stated that fragments located before the canal curvature have $100 \%$ success rate for retrieval, however, when fragments located at or beyond the curve the success rate decrease dramatically to $31 \%$.

Cone Beam Computed Tomography (CBCT) was used for its enhanced image offering multiple scans with different thickness. To ensure the standardization of readings multiple operators recorded the reading of the circumfrential dentin and mean value was calculated.

For the percentage of change of cross sectional area, the results were expressed in terms of relative rather than absolute values as the Relative Value is statistically more accurate and effective than the Absolute Value. The cross-sectional area of circumferential dentin reported a significant increase between pre and post operatively. For Protaper and WaveOne cross sectional areas of dentin at different levels in the 3 systems showed the highest cross sectional area at $7 \mathrm{~mm}$ followed by $5 \mathrm{~mm}$. Ultrasonics showed the least percentage of mean cross sectional 
area change in Protaper. WaveOne group needed more removal of dentin circumferentially to expose the broken fragment as it is only a single file system versus a Protaper full Sequence files. This came into agreement with Madarati et al ${ }^{(7)}$.

Amount of dentin removed was important to evaluate the ability of the tooth to regain its normal function after retrieval and to evaluate the ability to complete mechanical preparation, obturation without further complications as vertical root fracture.

Although previous investigators ${ }^{(31)}$ calculated the differences in root mass and canal volume before and after removal of the separated instrument, they draw the same conclusion with slight increase in the canal volume finding in the microtube samples over the Ultrasonics samples.

Regarding time elapsed till successful retrieval, ultrasonics were able to consume less time this came in agreement with Nagai et al ${ }^{(23)}$ stating that the ultrasonics retrieval time ranged from 3 to 40 minutes. While for iRS and microtube systems time ranged from 48 to 49 minutes in Protaper and WaveOne groups. This was in agreement with what was proposed by Terauchi et al ${ }^{(11)}$ that time needed for successful retrieval ranged from 2 to 40 minutes. Suter et al ${ }^{(32)}$ recommended that removal attempts of fractured instruments from root canals should not exceed 45 to 60 minutes. The increase in elapsed time for IRS and Microtube groups was attributed to the need for reusing ultrasonic to increase the space around the instrument head for the application of retrieval instrument which adversely affected the time and the amount of dentin removed circumferentially.

The results of iRS and Microtube systems recorded 20\%, 30\% perforation percentages respectively. This came in agreement with Alomairy $^{(22)}$ stating that 9 fragments out of 10 could not be retrieved without perforation occurred. The deeper the instrument was located at the root canal, the greater the risk of perforation ${ }^{(33)}$. Root perforations destroy the integrity of the root canal, thus adversely affecting tooth prognosis. Fu et al ${ }^{(24)}$ reported that the success rate for cases without perforation was $88 \%$ compared to $57 \%$ success rate for cases with perforations. Perforations were not the only complication that could be seen; unfortunately, tooth structure that have been removed during retrieval procedure might potentially lead to post treatment complications in the form of vertical root fracture, strip perforation, heat damages secondary to the use of Ultrasonics, and secondary fracture of the separated instrument.

On the basis of this study, there is no standardized system that could be used. Each individual case may require several approaches for removal. The results showed that removal can be successful in many cases. For the time being, Ultrasonics were found to be the most successful and universally used retrieval instrument. IRS, and microtubes need further assessment in straight canals as they were found to be unsuccessful in curved roots. Last, but not least, preserving dentin of the roots is of prime importance and clinicians' should not retrieve the fragment at the expenses of the survival of the tooth

\section{CONCLUSIONS}

- There is a difference in retrieval time between reciprocating and rotary instruments

- Percentage increase of cross sectional area is directly proportional with retrieval maneuvers

- Perforation incidences during retrieval procedures are sometimes non avoidable

\section{REFERENCES}

1. Choksi D, Idnani B, Kalaria D, Patel RN. Managment of an Intracanal Separated Instrument: A Case Report. I EJ 2013; 8: 205-7.

2. Ruddle C. Non Surgical Retreatment. J Endod 2004; 30: 827-45. 
3. Parashos P, Messer H. Rotary NiTi Fracture and its Consequences. J Endod 2006; 30: 722-5.

4. Gencoglu N., Helvacioglu, D. Comparison of the Different Techniques to Remove Fractured Endodontic Instruments from Root Canal system. Eur J dent. 2009; 3:90-5.

5. Schneider SW. A Comparison of Canal Preparations in Straight and Curved Canals. Oral Surg 1971; 32: 271-5.

6. Nevares G, Cunba R, Zuolo M, Bueno C. Success Rates for Removing or ByPassinng Fracture Instruments: A Prospective Clinical Study. J Endod 2012; 38: 442-4.

7. Madarati A A, Qualtrough AJE, Watts DC. Endodontists Experience Using Ultrasonic For Removal of Intracanal Fractured Instruments. Int Endod J 2010b; 43: 301-5.

8. Ruddle C. Non Surgical Retreatment. J Endod 2004; 30 : 827-45.

9. Shen Y, Coil JM, McLean AG, Hemerling DL, Haapasalo M. Defects in Nickel Titanium Instruments after Clincial Use: Part 5-Single Use From Endodontic Speciality Practices. J Endod 2009; 35: 1363-7.

10. Iqbal MK, Rafailov H, Kratchman SI, Karabucak B. A Comparison of Three Methods for Preparing Centered Platforms Around Separated Instruments in Curved Canals. J Endod 2006; 32: 48-51.

11. Terauchi Y, O’Leary L, Suda H. Removal of Separated Files from Root Canals with a New File- Removal System: Case Reports. J Endod 2006; 32:789-97.

12. Schrader C and Peters OA. Analysis of Torque and Force With Differently Tapered Rotary Endodontic Instruments In Vitro. J Endod 2005; 31[2]: 120-3.

13. Berutti E, Chiandussi G, Paolino DS, Scotti N, Cantatore G, Castelluci A. Canal shaping with waveone primary reciprocating files and protaper system: A comparative study, J Endod 2012; 38: 505-9.

14. Giuliani V, DiNasso L, Pace R, Pagavino G. Shaping Ability of WaveOne Primary Reciprocating Files and ProTaper System Used in Continuous and Reciprocating Motion. J Endod 2014: 1-4.

15. Capar ID, Ertas H, Ok E, Arslan H, Ertas ET. Comparative study of different novel nickel-titanium rotary systems for root canal preparation in severely curved root canals. J Endod 2014; $40:$ 852-6.

16. Gergi R, Osta N, Bourbouze G, Zgheib C, Naaman A. Effects of three nickel titanium instrument systems on root canal geometry assessed by micro-computed tomography. Int Endod J 2014; 1 : 1-9.
17. Ghodussi J, Bagherpour A, Mahmoudabadi F, Forghani M, Sarmad M. Residual Dentin Thickness of Bifurcated Maxillary Premolars Following Two Post Space Preparation Methods. Iran Endod J 2013; 8: 94-8.

18. Feldman G, Solomon C, Nnotaro P, Moskowitz E. Retrieving Broken Endodontic Instruments. J Am Dent Assoc 1974; 88: 588-91.

19. Nagai O, Tani N, Kayaba Y, Kodama S, Osada T. Ultrasonic Removal of Broken Instruments in Root Canals. Int Endod J 1986;19: 298-304.

20. Fu M, Zabang Z, Hou B. Removal of Broken Files by Using Ultrasonic Techniques Combined With Dental Microscope: A Retrospective Analysis of Treatment Outcome. J Endod 2011; 37: 619-22.

21. Cuje J, Bargholz C, Hulsmann M. The Outcome of Retained Instrument Removal in a Specialist Practice. Int Endod J 2010; 43: 545-54.

22. Alomairy K. Evaluating Two Techniques on Removal of Fractured Rotary NiTi Endodontic Instruments From Root Canals: An in Vitro Study. J Endod 2009; 35:559-62.

23. Plotino G, Pameijerc C, Grand N, Somma F. Ultrasonic in Endodontics: A Review of Literature. J Endod. 2007 Feb;33(2):81-95

24. Andrabi SM, Kumar A, Iftekhar H, Alam S. Retrieval of a Separated Nickel Titanium Instrument Using a Modified 18- Gauge Needle and CyanoAcrylate Glue: a Case Report. RDE2013; 4: 93-7.

25. Ingle J, Bakland I, Baumartner J. Ingle's Endodontics. 6 Hamilton, BC: Decker Inc; 2008

26. Chauhan R, Chandra A, Singh S. Retrieval of A Separated Instrument From The Root Canal Followed By NonSurgical Healing of a Large Periapical Lesion In Maxillary Incisors- A Case Report. Endod 2013; 25 : 68-73.

27. Hulsmann M, Schnikel I. Infleunce of Several Factors on Success and Failure of Removal of Fractured Instrument From Root Canal. Endod Dent Traumatol 1999; 15: 252-8.

28. Madarati A, Qualtrough A, Watts D. A MicroComputed Scanning Study of Root Canal Space: Changes After The Ultrasonic Removal of Fractured Files. J Endod 2009; 35: 125-8.

29. Suter B, Lussi A, Sequeira P. Probability of Removing Fractured Instruments from Root Canals. Int Endod J 2005; 38: 112-3.

30. Wilcox LR, Roskelley C, Sutton T. The Relationship of Root Canal Enlargment to Finger-Spreader Induced Vertical Root Fracture. J Endod 1997; 23: 533-4. 